\title{
Recent advances and future research directions in neurogastroenterology and endocrinology recommendations of the National Commission on Digestive Diseases
}

\author{
C. OWYANG
}

Division of Gastroenterology, Department of Internal Medicine, University of Michigan, Ann Arbor, MI, USA

\begin{abstract}
Recently, a draft of the report of the National Commission on Digestive Diseases was made available to the public. The Commission was given the task of assessing the current state of science in digestive diseases research, and developing a 10-year plan for digestive diseases research consistent with National Institutes of Health (NIH)'s mission of improving the health of the nation through research. Twelve topicspecific areas were selected for organizing the content of the long-range research plan. One chapter was devoted to Research on the Basic Biology of the Digestive System covering major biological pathways which regulate the physiology and biochemistry of the gastrointestinal tract. The author wrote about the areas related to neurogastroenterology, endocrinology and satiety. In this communication, recent advances in these areas are reviewed and major recommendations for future research endeavours are highlighted. Collectively, the recommendations will provide scientific direction for the NIH and all parties engaged in digestive disease research as they address opportunities in digestive diseases research over the next decade.
\end{abstract}

Keywords brain - gut axis, ENS, motility, NIH research funding.

Address for correspondence

Chung Owyang MD, Division of Gastroenterology, Department of Internal Medicine, University of Michigan, 3912

Taubman Center, SPC 5362, Ann Arbor, MI 48109-5362, USA.

Tel: +1 734936 4785; fax: +1 734936 7392;

e-mail: cowyang@umich.edu

Received: 18 July 2008

Accepted for publication: 9 September 2008

\section{GENESIS OF THE NATIONAL}

COMMISSION ON DIGESTIVE DISEASE AND ITS LONG RANGE RESEARCH PLAN FOR DIGESTIVE DISEASES

Decades of research in digestive disease have contributed to a significant reduction in the individual and national burden of many of these diseases. Despite these successes, the public cost of digestive diseases in the USA remains significant, and the sheer volume and scope of these illnesses highlight the crucial need for attention to these disorders through vigorous research efforts. In response to congressional report language accompanying the FY 2005 appropriation bills in the House and Senate for the Departments of Labor, Health and Human Services and Education and related agencies, on 26 July 2005, Elias A. Zerhouni, M.D., Director of the National Institute of Health, chartered the National Commission on Digestive Diseases. The Commission was given the task of assessing the state of the science in digestive diseases research and developing a 10-year plan for digestive disease research consistent with the National Institutes of Health (NIH) mission of improving the health of the nation through research. The Commission comprised 16 members who included academic researchers, medical professionals and patient advocates, who were appointed by the NIH Director after a public nomination process. Working groups composed of experts in diverse areas of digestive diseases research were formed to assist the Commission to identify major scientific advances and formulate highpriority research goals. Approximately six to 10 
members were chosen for each group based on expertise and knowledge of current research related to the working group topic. Twelve topic specific areas were selected for organizing content of the longrange research plan. One chapter was devoted to Research on the Basic Biology of the Digestive System covering major biological pathways, which regulate development, growth, digestion and metabolism, nutrient and fluid absorption and secretion, neurophysiology, endocrinology and satiety, microbiology and the microbiome and mucosal immunology. A draft of the Commission's report was recently made available to the public. Collectively, the Commission's recommendations will provide scientific direction for the NIH and all parties engaged in digestive disease research as they address opportunities in digestive diseases research over the next decade. The author wrote about the areas related to neurogastroenterology, endocrinology and satiety. In this communication, recent advances in the field are reviewed and major recommendations for future research endeavour are highlighted.

\section{LONG-RANGE RESEARCH GOALS AND RECOMMENDATIONS FOR FUTURE RESEARCH}

The Commission identified a number of long-range research goals and recommended them as high priority for future research. These are highlighted with comments from the authors as follows:

\section{(A) Define the basic cellular and molecular mechanisms responsible for neural activation, integration and regulation in the enteric nervous system}

The enteric nervous system (ENS) regulates motor patterns in the gastrointestinal (GI) tract. This division of the autonomic nervous system has been mapped extensively in a few animal models, but important mechanistic questions remain about the organization and function of enteric neurons and glial cells. Additional studies characterizing neural reflexes, neural plasticity, growth and development, and stem cell biology are needed to allow therapeutic control of enteric neural function.

\footnotetext{
A1. Objectives

- Understand the ionic and cellular regulatory mechanisms responsible for enteric nerve cell activation, synaptic transmission, integration and motor pattern development.
}

A1. Comments Remarkable progress has been made in the mapping of the ENS and how it is organized to achieve its role. ${ }^{1}$ Much is now known about the properties of individual neurons and how regional specialization occurs permitting differential functioning of the different regions of the GI tract. ${ }^{2}$ However, there is much to be learned about the ionic and cellular regulatory mechanisms responsible for enteric neuron activation, synaptic transmission, integration and motor pattern development. Two groups of enteric neurons deserve special attentions: the intrinsic primary afferent neurons (IPANS) and the interneurons. Because IPANs are the initiating neurons for enteric reflexes that control motility, blood flow and transepithelial movement of fluid in the gut, they are strategically placed to influence the intensity of the reflexes. Altered properties of IPANs could conceivably be involved in the development of intestinal hypersensitivity and altered motility in irritable bowel syndrome (IBS). ${ }^{3-5}$ Compounds that change the excitability of either the soma or the processes of IPANs or modify their response to neurotransmitters or inflammatory mediators may have therapeutic potential. Interesting targets include sites of sensory transduction $\left(\mathrm{P}^{2} \mathrm{X}^{6}\right.$ and $\left.5 \mathrm{HT}_{3}{ }^{7}\right)$ receptors and stretchactivated ion channels, ${ }^{8,9}$ ion channels which appear to be specific to IPANs (IK channels ${ }^{10}$ ), TTX-R Ina, NK3 receptors ${ }^{11}$ and receptors for inflammatory mediators such as cytokines. ${ }^{12-14}$

Interneurons mediate motility reflexes and are the sites of integration. It is important to identify the molecular and electrophysiological characteristics of these neurons and learn how they process information and coordinate sensory and motor reflexes. This is the first step towards identifying the distinct neural circuits involved in mediating different motility patterns. By defining the principle receptors, neurotransmitters and synaptic connections for each circuit we may be able to identify the 'switch' responsible to change one motility pattern to another. This kind of information may have important therapeutic implications.

\section{A2. Objectives}

- Characterize the molecular phenotypes of the different classes of enteric neurons (i.e. sensory, interneurons and motor neurons). Develop unique biomarkers to allow evaluation of the state of specific classes of enteric neurons during the development of GI motor disorders.

A2. Comments All enteric neurons can be placed in four functional classes based on their shapes, neuro- 
chemistries, function and cell physiological properties. ${ }^{1}$ These are IPANs, interneurons, motor neurons and intestinofugal neurons. These properties provide the different classes of enteric neurons with a neurochemical signature. These signature molecules include the primary transmitters of the neurons, the synthesizing enzymes for the transmitters, molecules that have transmitter or hormonal roles at other sites in the body, but are not necessarily transmitters of the enteric neurons that harbour them, and other markers such as calcium binding proteins. Thus, a chemical code can be found that defines enteric neurons and distinguishes neurons from each functional type. It is conceivable that this information may allow the scientists to develop unique biomarkers to identify the class of neurons which are malfunctioning during the development of GI motor disorders.

\section{A3. Objectives}

- Determine how enteric neurons function as a network to generate motor patterns, respond to luminal contents, produce stereotypical gut reflexes, generate sensory signals, and adapt to the conditions like inflammation or stress.

A3. Comments With better understanding of the roles of the various components of the ENS, we can begin to identify the distinct neural circuits involved in mediating different motility patterns. It is important to define the principle receptors, neurotransmitters and synaptic connections for each circuit and identify the 'switch' responsible to change one motility pattern to another. We need to examine how mechanical stimulation, metabolic stress and inflammation can alter these circuits. This type of information may be important to define the pathophysiology of IBS and various motility disorders. For reasons mentioned in Al, special emphasis should be placed on the IPANs and the interneurons. We need to characterize fully the plasticity of the channels, receptors and intracellular control systems of these neurons in response to inflammation, metabolic stress and changes in luminal bacteria flora. New technologies must be developed to visualize the state of activity of these neurons in live tissues and organs as they may help us to understand their role in initiating specific motility patterns.

\section{A4. Objectives \\ - Understand the development of the ENS and how adult neural stem cells might facilitate the repair of a defective ENS resulting from developmental defects or pathophysiological damage.}

A4. Comments Until recently, the postnatal peripheral nervous system was thought to lack stem cells. A technological breakthrough occurred in 2002. Using flow cytometry, the Morrison laboratory was able to identify prospectively and isolate the neural crest stem cells (NCSCs) that are capable of forming multilineage colonies in culture. ${ }^{15,16}$ These investigators showed that NCSCs persist in the adult gut and undergo changes in self renewal. These exciting new findings open up a new possibility for regeneration after injury or disease. Currently, much remains to be learned about how the various signals interact to yield a 'normal ENS'. We should continue to search for clue as we begin to identify the developmental defects resulting in neuronal loss such as in Hirschsprung's disease and hypertrophic pyloric stenosis. We need to identify the molecules and pathways that promote proliferation and differentiation of enteric neurons and/or guide the growth of enteric axons to their target.

\section{A5. Objectives}

- Understand the role of glial cells in maintaining the structure and integrity of enteric ganglia and in regulating the functions and health of enteric neurons.

A5. Comments The enteric neurons and nerve fibre tracts are supported by numerous glial cells. In the central nervous system (CNS), glial cells have an important role in synaptic transmission, plasticity and immunoprotection. Glial-derived neurotrophins and neurotrophic factors such as glial-derived neurotrophic factor (GDNF) have strong effects on neurite outgrowth and differentiation and are able to protect neurons from apoptosis under various inflammatory conditions. It is likely that glial cells may play similar roles in the ENS. This needs to be thoroughly investigated. It was reported that GDNF availability determines enteric neuron numbers by controlling ENS precursor proliferation. ${ }^{17} \mathrm{GDNF}^{-/-}$mice have hypoganglionosis in the stomach and aganglionosis of the small bowel and colon. ${ }^{18,19}$ It is conceivable that some of the motility disorders secondary to degenerative loss of enteric neurons may be due to structural and/or functional abnormality of the glial cells. Glial cells also play the important roles in the maintenance of gut mucosal integrity. ${ }^{20,21}$ In human Crohn's disease and experimental colitis in rats, the GDNF is upregulated. ${ }^{22}$ This neurotrophic factor has strong anti-apoptotic effects on colonic epithelial cells which may be responsible for its protective action on the epithelial lining during mucosal inflammation. ${ }^{22}$ The mechanisms responsible for upregulation of the GDNF in inflammatory bowel conditions should be examined and its cellular actions 
to promote epithelial restitution should be investigated. Alteration of this novel gut neuroepithelial cross-talk may contribute to a more serve course of inflammatory bowel disease (IBD).

\section{A6. Objectives \\ - Understand how inflammatory cells and media- tors influence neural activation and integration.}

A6. Comments Inflammation has widespread effects on neural activation and integration. The excitability and synaptic transmission of both the $\mathrm{AH}$ - and S-type submucosal neurons are increased in experimental colitis in guinea pig. ${ }^{23}$ The molecular mechanism responsible for the activation of the intrinsic sensory neurons during inflammation is not known and may involve plasticity of receptors, ion channels and intracellular signalling molecules. This is an important area for research as inflammation may alter sensory perception and activate neural reflex pathways resulting in abnormal motility. Inflammation seems to have a differential effect on motor neurons in the GI tract, as the packaging, storage, and release of neurotransmitters from the motor neurons and sympathetic nerve endings in the myenteric plexes are impaired. ${ }^{24,25}$ This is mediated by inteleukin- $1 \beta{ }^{26}$ Impaired function of the motor neurons in the ENS may result in abnormal lower oesophageal sphincter (LOS) function and GI dysmotility. Enteric neurons are not just bystanders during inflammation; they may actively participate in the inflammatory response. Cytokines enhance the release of substance $\mathrm{P}$ from sensory neurons and substance $\mathrm{P}$ in turn activates immune cells and modulates the inflammatory response. Furthermore, the bioavailability of substance $\mathrm{P}$ is increased in the inflamed gut because of the suppression of the tissue level of neural endopeptidase. ${ }^{27}$ Thus the multiple levels of cross-talk between enteric neurons and immune cells may set up a vicious cycle perpetuating the pathogenesis of chronic pain. It is conceivable that a greater understanding of the mechanisms responsible for neuroplasticity in enteric neurons during inflammation may reveal new and effective approaches to treatment.

\section{(B) Understand the structure, function and regulatory mechanisms responsible for motility in the GI tract}

The ENS exerts control over smooth muscle cells to develop patterns of contractile responses, such as peristalsis, segmentation, tonic contraction, retroperistalsis and others. ENS control is superimposed upon spontaneous activity of the musculature (so-called 'myogenic' activity of smooth muscle tissues) and contributions from a variety of additional regulatory systems. A more complete understanding is needed of the interactions of the ENS with smooth muscle cells and the mechanisms responsible for regulation of contractile behaviour to develop the targeted therapies to improve motor function.

\section{B1. Objectives}

- Understand the molecular signalling pathways responsible for generation of tonic and phasic contractions in GI muscles and how these pathways are altered in pathophysiological conditions.

B1. Comments Gastrointestinal smooth muscle exhibits variable tone, on which are superimposed rhythmic contractions driven by electrical slow waves. The physical characteristics of these two types of contractions and their spatiotemporal patterns, function and regulatory mechanisms are markedly different from each other. The slow waves initiated from the interstitial cells of Cajal (ICC) are mainly responsible for the initiation of phasic contractions of the smooth muscle. $\mathrm{Ca}^{2+}$ influx during the depolarization phase of each slow wave triggers a transient contraction. Phosphorylation of the $20-\mathrm{kDa}$ regulatory myosin light chain $\left(\mathrm{MLC}_{20}\right)$ is a prerequisite of smooth muscle contraction. $\mathrm{MLC}_{20}$ phosphorylation during the initial transient contraction induced by an agonist is mediated by $\mathrm{Ca}^{2+} /$ calmodulin dependent MLCK, whereas $\mathrm{MLC}_{20}$ phosphorylation during the subsequent sustained contraction is mediated by $\mathrm{Ca}^{2+}$-dependent MLCK and inhibition of MLCP. Multiple signal transduction pathways and molecules are involved in the mediation of muscle contraction depending on the regions of the GI tract and the types of contractions. Many of these signalling molecules may be affected by pathological conditions such as inflammation. For example IL- $1 \beta$ released during inflammation has been shown to enhance the expression of RGS4 ${ }^{28}$ which in turn accelerates the deactivation of $\mathrm{G}_{\alpha \mathrm{qq}}$, leading to a decrease in phosphoinositide hydrolysis and IP3dependent $\mathrm{Ca}^{2+}$ release and the inhibition of initial contraction and $\mathrm{MLC}_{20}$ phosphorylation. ${ }^{29}$ Similarly, ageing and metabolic disorders such as diabetes can also alter the expression of key signalling molecules which regulate muscle contraction. Studies of this kind may help to identify therapeutic targets for prevention and/or intervention.

\section{B2. Objectives \\ - Clearly define the role and mechanisms of electro- mechanical and pharmaco-mechanical coupling in}


generating tone and phasic contractions in GI muscles and the effects of sex hormones, inflammatory factors and ageing on these mechanisms.

B2. Comments The ratios of the activities of MLCK and MLCP determine the net phosphorylation of $\mathrm{MLC}_{20}$ and this in turn regulate muscle contraction. The activities of MLCK and MLCP are regulated by electromechanical and pharmacomechanical couplings. Electromechanical coupling is caused by an increase in cytosolic free $\mathrm{Ca}^{2+}$ by membrane depolarization and influx through membrane calcium channels. Abnormalities in slow-wave generation or propagation or smooth muscle $\mathrm{Ca}^{2+}$ channels may lead to defective electromechanical coupling. The pharmamechanical coupling is caused by the activation of cell signalling pathways by the binding of ligands to their membrane receptors, followed by the activation of kinases that modulate the activity of MLCP or MLCK. Pharmacomechanical coupling increases $\left[\mathrm{Ca}^{2+}\right] \mathrm{i}$ by the opening of receptor-operated $\mathrm{Ca}^{2+}$ channels through non-selective cationic channels, and by release of $\mathrm{Ca}^{2+}$ from the intracellular stores to enhance the phosphorylation of $\mathrm{MLC}_{20}$ by MLCK. Different contribution of electromechanical and pharmacomechanical couplings in response to an agonists will determine the type of muscle contraction. These are important mechanisms for determining the contractile performance of smooth muscle cells and they can be affected by sex hormones, inflammatory factors and ageing. This is an important area for future research.

\section{B3. Objectives}

- Determine the molecular basis for electrical coupling between GI smooth muscle cells and between smooth muscle cells and ICC and the consequences of a breakdown in electrical coupling on contractile behaviour.

B3. Comments The relationships between electrical events in ICC and in smooth muscle cells have been extensively investigated. The ICC form gap junctions with each other and with smooth muscle cells; these junctions provide structural basis for electrical coupling between these cells. Slow-wave activity is generated and actively propagated within the ICC networks, and slow waves passively spread to neighbouring smooth muscle cells. ${ }^{30}$ Thus, the loss of slow waves due to damage of ICC in any parts of the GI tract might adversely affect its motor performance. Pacemaker ICC possess distinct channels responsible for initiation of slow waves and their propagation to electrically coupled smooth muscle cells. The mecha- nism appears to involve a localized release in $\left[\mathrm{Ca}^{2+}\right] \mathrm{i}$ that causes the opening of non-selective cationic channels in ICC. ${ }^{31}$ The precise trigger for $\mathrm{Ca}^{2+}$ release via $\mathrm{IP}_{3}$ receptors in the absence of agonists is unknown. This is an important area for further investigation as the regulation of the open probability of IP3 receptor in ICC is central to the coordination of pacemaker activity. Furthermore, little is known about voltagedependent regulation of $\mathrm{IP}_{3}$ production or another second messenger that increases the sensitivity of PLC or other pathways in the ICC. In addition, the molecular entity responsible for the dihydropyridine-resistant channels in ICC has not yet been identified. This type of information will shed light on the mechanisms responsible for the generation and propagation of slow waves in the ICC. Abnormalities in these regulatory pathways may conceivably lead to motility disorders.

\section{B4. Objectives}

- Determine the mechanisms and the role of calcium sensitization in response to neurotransmitters, hormones and paracrine substances in GI contractile behaviour and whether this mechanism is altered by inflammation, sex hormones, or ageing.

B4. Comments $\mathrm{Ca}^{2+}$ channels and $\mathrm{Ca}^{2+}$-activated $\mathrm{K}^{+}$ channels are the electrical apparatus that sustains rhythmicity in smooth muscle. The $\mathrm{Ca}^{2+}$ sensitivity of $\mathrm{K}^{+}$channels links their activity to that of $\mathrm{Ca}^{2+}$ channels and creates the dynamic framework for rhythmic electrical activity. Excitatory neurotransmitters or hormones may increase intracellular $\mathrm{Ca}^{2+}$ by inducing $\mathrm{Ca}^{2+}$ influx and mobilizing intracellular $\mathrm{Ca}^{2+}$ store resulting in contraction. On the other hand, inhibitory neurotransmitters act through protein kinase $\mathrm{A}$ or protein kinase $\mathrm{G}$ to decrease $\left[\mathrm{Ca}^{2+}\right]$ i. They do so by inhibiting $\mathrm{Ca}^{2+}$ release and enhancing sequestration of $\mathrm{Ca}^{2+}$ in intracellular store, mechanisms that are well suited to the relaxation of tonic contraction, or by hyperpolarizing the plasma membrane during slowwave activity and reducing $\mathrm{Ca}^{2+}$ influx, a mechanism well suited to the relaxation of phasic contraction. The hyperpolarization determines the profile or occurrence of phasic contractions. It is important to determine the molecular mechanisms responsible for calcium sensitization as this process can be affected by disease conditions such as inflammation. Inflammation suppresses the $\mathrm{Ca}^{2+}$ current through L-type $\mathrm{Ca}^{2+}$ channels (Cav 1.2). ${ }^{32}$ This is mediated by the suppression of the pore forming $\alpha \mathrm{IC}$ subunit of Cav 1.2 channels. $^{32}$ Molecular studies of this kind are important to understand how excitation-contraction coupling may be altered in disease process. 


\section{B5. Objectives}

- Determine the basis for spontaneous electrical activity in smooth muscle cells and tissues and the mechanisms for propagation of electrical activity in the generation of motor behaviour of GI organs.

B5. Comments The smooth muscle cell response to electrical inputs from ICC underlies the motility patterns in GI organs. Slow waves produce a cycle of depolarization and repolarization that conducts to smooth muscle cells and changes the open probabilities of the many voltage-dependent ion channels expressed by smooth muscle cells. Voltage-dependent ion channels form the backbone of the smooth muscle response to electrical rhythmicity, but metabotrophic and $\mathrm{Ca}^{2+}$-dependent ion channels also contribute to the integrated responses of these cells. Responses are further tuned to create the observed motility patterns by paracrine, hormonal and neural inputs to the smooth muscle/ICC syncytium. Many of the stereotypical motility responses of these cells also depend on modulations of ion channels. Further studies should emphasize on characterizing the variety and complexity of ion channel expression in GI smooth muscle and how these elements can be affected by inflammation, sex hormone or ageing.

\section{B6. Objectives}

- Understand stretch-dependent mechanisms that regulate the excitability of GI smooth muscles and contribute to patterns such as receptive relaxation, peristalsis and gut stasis.

B6. Comments Recent studies indicate that ICC mediate mechanosensitive response in the GI tract. ${ }^{33}$ This may be achieved by physical transfer of mechanical information at specialized junctions known as seg and socket junctions. ${ }^{33}$ Alternatively, the mechanical force may be transmitted through the production of prostaglandins. ${ }^{33}$ Slow waves conduct via low-resistance junctions to smooth muscle cells and decay as a function of distance from the ICC network. The rate of decay is dependent on the cable properties of the smooth muscle syncytium. These are modulated via the plethora of ionic conductances expressed by smooth muscle cells. The molecular mechanism regulating these conductances is an important area of investigation as they tune the smooth muscle response. Oscillation of membrane potential through the slow-wave cycle results in periods of high and low open probability for $\mathrm{Ca}^{2+}$ channels, and this organizes the contractile pattern into a series of phasic contractions contributing to motility patterns such as peristalsis and segmentation. The force and pattern of phasic contractions depend on coordination from the ENS. Neural inputs set the excitability of the smooth muscle cells by regulating resting membrane potential or by altering the responses of voltage-dependent channels to slow-wave depolarizations. Superimposed on neural regulation are multiple layers of additional regulation caused by circulating hormones and paracrine substances. Thus, the final outputs of the smooth muscle contractions and GI motility are a highly complex, integrated response involving multiple cell types and multiple regulatory levels. Abnormality in any level of regulation may result in impaired motility. Investigation in this area is likely to shed new light on the pathogenesis of various motility disorders.

\section{B7. Objectives}

- Determine the effects of inflammatory mediators on the structure, function and phenotype of GI smooth muscle cells and ICC.

B7. Comments Inflammation has widespread effects on the structure and function of smooth muscle cells and ICC. Through the release of cytokines and other inflammatory agents, inflammation may have differential effects on the expression of receptors, G proteins, PKCs or ion channels. The genomic effects on those key signalling molecules may result in altered excitation-contraction coupling and muscle contractile pattern. Inflammation also significantly affects the structure and function of ICC. The density of ICC is reduced in the inflamed gut and their processes are damaged. ${ }^{34}$ At the same time, it reduces the amplitude and duration of slow wave. This directly contributes to a smaller increase in $\left[\mathrm{Ca}^{2+}\right] \mathrm{i}$ and reduction phasic contractions. The smooth muscle cells are not only targets of inflammatory mediators but also secrete inflammatory mediators. For example, acid can activate vanilloid receptors (TRPV1) present on the squamous epithelial cells that release platelet activating factor. ${ }^{35}$ This may increase reactive oxygen species generation in smooth muscle cells and in turn impair the LOS tonic contraction. Catalase treatment restores the weak tonic contraction of circular muscle from a human donor with erosive oesophagitis. The expression and secretion of inflammatory mediators by colonic smooth muscle cells may also be the basis of persistence of motility dysfunction in patients with IBD in remission and in patients with postinfectious IBS. Hence, research in myoneuroimmune interaction has important clinical and therapeutic implications. 


\section{(C) Develop research tools to investigate the structure and functional organization of the ENS}

The multiple constituents of the ENS are characterized by a dynamic cross-talk between the enteric neurons, glial cells, ICC, smooth muscle cells and enteroendocrine cells. Hence, functional and structural studies need to be performed with tools that allow dynamic visualization of the activity of the relevant cells in situ. Characterization of receptors, channels and signal transduction systems unique to different cell types and how they interact with luminal events are critical to understanding how information is processed in the coordination of motility, secretion and absorption.

\section{C1. Objectives}

- Investigate molecular and electrophysiological characteristics of various ENS cellular components, which may be targets for new developments to treat motility disorders.

C1. Comments The structural and functional organization of ENS continues to evolve. We need to more fully characterize the enteric neural networks; understand the cross-talk between the enteric neurons, glial cells, ICC, smooth muscle cells and enteroendocrine cells; and define the signal processing within the ENS in response to nutrient, mechanical stimulation, inflammation, metabolic stress and changes in bacterial flora. We must investigate the molecular and electrophysiological characteristics of the various cellular components of the ENS. This includes identification of receptors, channels and signal transduction systems unique to different cell types in the ENS as they may be targets for new drug developments to treat motility disorders.

\section{C2. Objectives}

- Develop tools to visualize the state of activity of relevant cells in live tissues, organs and systems.

C2. Comments The multiple constituents of the ENS can have profound effects on its functioning. In addition to the nerves and smooth muscle cells, the normal functioning of the system requires participation of the ICC, glial cells and enteroendocrine cells. For example, research on the ICC has dramatically altered the way we look at regulation of smooth muscle. Interstitial cells of Cajal pace GI muscle by initiating slow waves in both muscle layers and appear to be the preferred sites for reception of neurotransmitters, especially for acetylcholine and nitric oxide. ${ }^{2}$ Technically, we must develop the tools to visualize state of activity of rele- vant cells (ICC, enteric neurons, muscle cells and neuroendocrine cells) in a live context of tissue (in vitro or in vivo), organ and system (in vivo) and develop quantitative analysis of spatio-temporal patterns. Ultimately, this may help to elucidate the roles of various cellular components of the ENS in the mediation of physiological events such as motility, sensory transmission, secretion and blood flow. There is a need to integrate cellular events with whole system physiology.

\section{C3. Objectives}

- Characterize alterations in gut-based 5HT and corticotropin-releasing factor (CRF) in IBS and motility disorders, including genetic polymorphisms affecting ligands, receptors, transporters, enzymes and/or signalling systems.

C3. Comments The recent characterization of the peripheral serotonin/5HT receptor signalling system in the ENS represents a major advance in our endeavour to treat functional bowel disorder. ${ }^{36}$ Multiple 5HT receptor subtypes $(5 \mathrm{HT} 1,5 \mathrm{HT} 2,5 \mathrm{HT} 3$ and $5 \mathrm{HT} 4)$ are present in the ENS. They mediate neuronal excitability and synaptic transmission. The differential distributions of 5HT subtypes ${ }^{36-39}$ make it possible to use 5HT antagonists and agonists to treat motility disorder and IBS. The serotonergic network should be an important target for new therapeutic approaches.

Given the importance of the peripheral 5HT signalling system in GI motor and sensory disorders, we need to continue our efforts to characterize the alterations in the gut-based 5HT signalling system in IBS and motility disorders. This may include SERT, modulators of SERT expression, and a number of enterochromaffin cells and contents. The mechanism responsible for diminished serotonergic signalling in inflammation should be examined. Transcripts encoding tryptophan hydroxylase-1 and SERT can be decreased. Successive potentiation of $5 \mathrm{HT}$ and/or desensitization of its receptor could account for the symptoms observed in diarrhoea-predominant and constipation-predominant IBS respectively. This possibility should be investigated. Genetic polymorphisms affecting 5HT synthesis, receptors, transporters and/or signalling systems may contribute to the development of IBS. This is an important area of future investigation.

The CRF signalling pathway coordinates endocrine, behavioural and immune responses to stress. ${ }^{40}$ The Activation of CRF receptor 1 and 2 produces differential effects on gastric and colonic motility. ${ }^{41-44}$ Clinical studies show that stimulation of CRF1 pathways produces the key symptoms of IBS diarrhoea-predom- 
inant patients. ${ }^{45}$ These symptoms are alleviated by CRF1 receptor antagonists supporting the involvement of the CRF1 system at the central and peripheral sites in the pathogenesis of IBS triggered by stress. ${ }^{45}$ The CRF1 receptor antagonist that is directed at normalizing a sensitized CRF system holds great promise for a variety of stress-related GI disorders including IBS and cyclical vomiting syndrome. ${ }^{46}$ Further efforts should be directed at investigating possible abnormalities of the CRF signalling pathway in IBS patients and defining the subtypes of IBS patients whom are most likely to respond to treatment with CRF1 receptor antagonist.

\section{C4. Objectives}

- Identify molecules and pathways that promote proliferation and differentiation of enteric neurons and/or molecules responsible for directing enteric axons to their targets. Define the molecular basis for chemo- and mechano-receptors in the gut to sense ingested nutrient environment and gain better understanding about interactions between nutrient and microbe-sensing mechanisms in the gut.

C4. Comments Research to elucidate the events in early ontogeny will continue to provide important clues on the mechanism underlying ENS formation. These studies revealed an ever increasing range of molecules essential for the normal development of the ENS. The RET/GFR $\alpha 1 /$ GDNF signalling pathway plays a number of key roles during ENS development, including the promotion of survival and proliferation of enteric precursors within the gut and their subsequent differentiation into enteric neurons. ${ }^{4-50}$ In addition GDNF is also a potent chemoattractant for neural crest cell-derived enteric precursor cells. ${ }^{51}$ Perhaps the most novel aspect of RET biology is that it induces 'positive' survival signals in the presence of its ligand GDNF, and 'negative' cell death signals in the absence of GDNF. ${ }^{52-53}$ This may provide an alternate mechanism for the pathogenesis of Hirschsprung's disease and other motility disorders due to degeneration of the ENS. The EDNRB/EDN 3 system provides another critical signalling pathway necessary for ENS development. Its activation enables colonization of the distal hindgut. ${ }^{54}$ The EDNRB/EDN 3 signalling system is also important in the maintenance of the neural crest cells in a multipotential undifferentiated progenitor-like state. ${ }^{55}$ This type of studies is important as they may shed light on how adult neural stem cells might facilitate repair of a defective ENS resulting from developmental defects or pathophysiological damage.
On a separate subject, there is increased understanding of the biophysical principles of sensory function in the GI tract. This has important pathophysiological and therapeutic importance in a number of motility conditions including visceral hypersensitivity and IBS. Information on contractile state of the musculature and distension of the gut wall is coded by mechanoreceptors which include both low and high threshold mechanoreceptors. The electrophysiological properties of these receptors may be altered by stress or inflammatory conditions. Furthermore, there is a third group of mechanoreceptors known as silent nociceptors which can be sensitized by inflammatory mediators resulting in spontaneous firing and responses to normally innocuous mechanical distension. The molecular mechanism responsible for the plasticity of these receptors is an important area for future research. Sensory transduction of chemical information about luminal nutrient concentrations in the small bowel involves paracrine signalling from enteroendocrine cells to spinal and vagal afferent terminals in the gut wall. The Activation of enteroendocrine cells can be accomplished by mechanisms resembling oral taste sensation. Several enteroendocrine cell types throughout the gut express T1R $2 / 3$ sweet taste receptors, T2R-family bitter

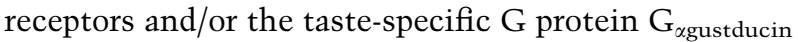
and these cells are activated by tastant molecules. ${ }^{56-60}$ This line of research may be exploited for new drug development. For example, intestinal L cells, which secrete GLP1, oxyntomodulin and peptide YY (PYY), express a pathway for nutrient sensing and signal transduction previously believed to be limited to tastebud cells. ${ }^{60}$ Because the details of sweet taste receptor activation are understood at the atomic level, one can imagine rational design of non-caloric artificial sweeteners optimized for the combination of palatable taste and potent L-cell activation. These might supplement treatment of obesity and diabetes. ${ }^{61}$

\section{(D) Characterize the neuromuscular phenotypes of human GI tissues}

Much has been learned in recent decades about the organization of the ENS and cellular mechanisms involved in generating normal gut motility patterns. Most information has come from studies of laboratory animals. It is important to translate this information into studies of human GI muscles to determine the neuromuscular phenotypes driving normal human GI motility. Although access to human samples is limited, surgery for GI conditions like cancer or obesity presents an opportunity for studying human physiology. 


\section{D1. Objectives}

- Understand the excitability and contractility mechanisms in human GI muscles. Translate knowledge obtained from animal models to human physiology.

D1. Comments The physiology of the ENS and the organization of the control circuits have mostly been studied in the guinea pig. Although pharmacological investigations of neurotransmission and immunohistochemical studies innervation patterns suggest that the enteric neurons in humans are similar to those of other mammals, detailed information about functional characteristics of these neurons in humans remain largely unknown. This is especially true about channel properties, signal pathways, and electrophysiology of these neurons as they require fresh human tissues for investigation and this is not readily available. Animal models continue to be useful to provide important information on the organization of the ENS but there are differences between species such as the arrangement of ganglia and the chemical coding of enteric neurons. This is critical information to obtain before we can translate knowledge obtained in animal models to human physiology.

\section{D2. Objectives}

- Understand the structure and function of the human ENS. Determine which animal models best simulate the integration and cellular phenotypes of human enteric neurons.

D2. Comments Essentially, all functional types of enteric neurons have been characterized by their shapes, neurochemistries and cell physiological properties in the guinea pig and more recently in their orthologs in human and several other species. These neurons can be placed in four functional classes, IPANs, interneurons, motor neurons and intestinofugal neurons. Pharmacological investigations of neurotransmission and immunohistochemical studies indicate that muscle motor neurons and secretomotor neurons in humans are similar to those in other mammals, and that human equivalents of IPANs have been identified. ${ }^{1}$ The interneurons are more difficult to study but current information suggests that they are similar to those of other species. As documented in A2, the shapes, neurochemistries, function and cell physiology properties provide the different classes of enteric neurons with a neurochemical signature. This information conceivably may allow us to develop unique biomarkers to identify the class of neurons which are malfunctioning in GI motility disorders.
Hence, an important task will be to determine the chemical identifiers of each class of the functionally defined neurons in the human ENS. This will be important for future investigations of enteric neuropathology.

\section{D3. Objectives}

- Characterize motor innervation of the human muscularis and identify the major neurotransmitters, the cells that are innervated by motor neurons, and the mechanisms of postiunctional neural responses.

D3. Comments The motor neurons of the ENS in humans appear to be analogous to those in other mammals, the excitatory motor neurons being Dogiel type I cholinergic neurons with tachykinin immunoreactivity and the inhibitory motor neurons being anally projecting Dogiel type I neurons with nitric oxide synthase and vasoactive intestinal polypeptide (VIP) immunoreactivity. These deductions are supported by pharmacological investigations of enteric reflexes in human

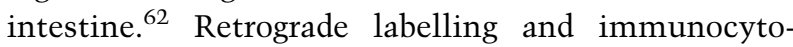
chemistry studies suggest that VIP-containing secretomotor neurons occur in human intestine. Most of these neurons also contain acetyl choline as well. In the colon, VIP and acetylcholine are found in distinct enteric neurons. ${ }^{63}$ The functional significance of these regional differences in the neurontransmitter contents of human motor neurons requires further investigation. Apart from supplying smooth muscle and secretory epithelium, motor neurons may also supply blood cells and innervate entero-endocrine cells. In human, motor neurons are mono-axonal, and almost all have S-type electrophysiological properties. Studies on the structural and functional characteristics of motor neurons in human are of great clinical importance as many of the GI dysmotility disorders may be traced to malfunction of the motor neurons within the ENS.

\section{D4. Objectives}

- Define the receptors and signalling pathways that are involved in neural, hormonal and paracrine regulation of human GI muscle function.

D4. Comments Multiple receptors and signal transduction pathways exist in smooth muscle cells and ICC. They mediate and condition the response of smooth muscles to slow-wave depolarizations and humoral substances like hormones, paracrine substance and inflammatory agents. The sites of action of some of these bioactive substances may also be at ICC, but the importance of ICC as targets for these agents in human is poorly understood. Several motility disorders 
have been associated with loss of ICC, thus investigation of these cells in humans may shed light on the pathogenesis of some of the motility disorders. Furthermore, alteration in receptor numbers or affinity or signal transduction pathways may occur in smooth muscle cells or ICC in response to inflammation or metabolic stress. This is an important area of investigation in human motility disorders.

\section{D5. Objectives}

- Develop methods of organ or cell culture that preserve the phenotypes of human muscle cell components. Determine methods to culture smooth muscle cells, ICC, enteric neurons and other cellular components without dramatic changes in the native phenotype.

D5. Comments As access to human samples is always going to be limited, primary cells derived from humans with defined attribute would enable in vitro studies to characterize the signalling pathways of various cell types found in the ENS. Important cell lines to be defined and established include NCSCs, progenitors of ICC, glial cells and specific native neurons and enteroendocrine cells. To do so, it will be important to develop better cell specific markers. In addition, innovative techniques are required for the long-term maintenance of cells in culture under circumstances in which cells do not undergo major change in their characteristics. Moreover, central facilities to acquire and maintain these cells for distribution would be helpful.

\section{(E) Integrate cellular events in ENS with whole system physiology and translate findings to pathophysiological conditions}

Normal functioning of the GI tract requires different components of the ENS to operate in unison, emphasizing the importance of identifying relationships in an organismal context. Advances in neurobiology of brain-gut interaction, together with availability of new neuroimaging modalities, greatly enhance our ability to study functional GI disorders and search for new therapeutic targets. Recognition of the GI tract's crucial role in satiety signalling and control of energy homeostasis, body weight and various metabolic systems provides a new framework to study the disorders of energy homeostasis. Pathophysiological models coupled with genomic analysis offer new opportunities to discover molecular mechanisms responsible for agerelated neuron degeneration and provide avenues to reconstitute the ENS networks in diseased organs.

\section{E1. Objectives}

- Identify distinct brain circuits responsible for various gut functions and pain perception and characterize the signalling systems and receptors within these neural circuits using PET ligand imaging in rodent models and humans with IBS and functional dyspepsia.

E1. Comments Major advances have been made in our ability to image the living human brain with various neuroimaging modalities. ${ }^{64}$ This has greatly enhanced our ability to study brain-gut interactions in health and in disease conditions. Symptom components of IBS can be dissected and attributed to specific areas of the brain that mediate cognitive, emotional and motivational component of the discomfort. ${ }^{64,65}$ This new approach to the study of functional GI disorders provides more insightful information on the pathophysiology of this group of disorders. Efforts to characterize the signalling systems and receptors in these neural circuits using PET ligand imaging in rodent models and humans may provide novel therapeutic targets for rationale design of new drugs to treat IBS and functional dyspepsia. Furthermore, the ability to study a neurobiological substrate with imaging modalities rather than relying on highly variable subjective symptoms (e.g. Rome criteria) makes it possible to investigate the role of genetic factors ${ }^{66}$ and receptor physiology $^{67}$ on the pathophysiology of symptoms. We should have a precise endpoint to evaluate therapeutic interventions on distinct brain networks involved in afferent processing and modulation. Meaningful results from such studies can be obtained from much smaller samples of subjects compared with epidemiological or traditional pharmacological studies.

\section{E2. Objectives}

- Develop contemporary techniques for probing genetic and proteomic changes that occur with age. Establish the mechanism that maintains the integrity of the ENS and its capacity to respond to altered function or 'plasticity' in adulthood and old age.

E2. Comments Degenerative and developmental loss of enteric neurons continues to be the main cause of severe motility disorders. We need to have a better understanding of the molecular mechanisms underlying age-related apoptosis of ENS neurons. Contemporary techniques for probing genetic and proteomic changes that occur with age, such as the use of oligonucleotide microarray and protein chip technologies, will help the field to evolve from phenomenology to hypothesis-driven research. It is important to establish the mecha- 
nisms that maintain the integrity of the adult ENS and its capacity to respond to altered function or 'plasticity' in adulthood and old age. Studies of neurotrophic and growth factors and the associated signalling pathways may provide some clues for this type of investigation.

\section{E3. Objectives}

- Investigate the cellular and molecular mechanisms of neural and endocrine bi-directional communication between the gut and the CNS for regulation of weight and metabolic function and the associated neurohumoral events.

E3. Comments Obesity research remains the top priority for investigators in the United States. The capacity to adjust food intake in response to changing energy requirements is essential for survival and good health. We need to define the diverse blood-borne and affective neural signals that transmit information regarding nutrient status and energy stores to the brain where it is integrated with cognitive, visual, olfactory and taste cues. Understanding the complexity of this energy homeostasis system will then allow us to start to search for mutation of key molecules mediating these pathways in patients with severe obesity.

Currently, little is known about the site and mechanisms by which alterations in blood glucose levels and other nutrients are sensed. Comprehensive studies must be performed to investigate the peripheral mechanisms of nutrients sensing. The sites are likely to involve the CNS as well as the vagal afferent fibres that may mediate gastric motility, satiety and hepatic glucose uptake and outputs. Abnormality of this pathway may occur in patients with diabetes and/or obesity.

Further studies are needed to understand the cellular and molecular mechanisms of neuro and endocrine bidirectional communication between the GI tract and CNS to regulate body weight and metabolic function. Most of the satiation and orexigenic peptides are found in the GI tract. ${ }^{68}$ These include ghrelin, GLP1, PYY, oxyntomodulin and urocortin. Rationale manipulation of the neuroendocrine pathways regulating apeptide may be used to treat obesity. For example, bariatric surgery works primarily by physiological rather than mechanical mechanisms. A better understanding of the physiological and molecular mechanisms by which bariatric surgery improves body weight and metabolic regulation might shed light on novel mechanisms we can manipulate to treat obesity.

\section{E4. Objectives \\ - Develop suitable animal models to mimic diseases of the ENS.}

E4. Comments Progress in the research of functional bowel disease is partly hampered by lack of suitable animal models to mimic the disease conditions. Although animal models of visceral hypersensitivity are available, they are not ideal as the pathophysiology may be quite different. We need to establish scientific criteria to determine the validity of animal models for IBS and other motility disorders. Furthermore, the current range of animal models for studying motility disorders and functional bowel diseases could be expanded by the development of tools that permit targeted genetic studies in various animal models within specific tissues and cell types of the ENS. Validation of specific promoters and/or minigenes that drive tissue- or segment-specific gene expression in mouse and other relevant models is required. Efforts to strengthen neurogastroenterology research across the scientific community might include: (i) establishment of a coordinated system that would allow for the identification, characterization and distribution of suitable mouse lines in a defined background, including conditional gain and loss of function alleles that can be induced in a temporally regulated manner and (ii) develop centralized animal resources to serve as a repository of useful models to facilitate research on molecular pathophysiology of disease development.

\section{(F) Translate knowledge of the ENS in digestive health and disease into diagnostics and therapies for human disease}

Research on the pathophysiology of human motility disorders needs to be aggressively translated into the treatment of human diseases. This includes advances in neuroimaging modalities to study brain-gut interactions, efforts to unravel the complexity of energy homeostasis systems, an understanding of some of the key neural circuits in the ENS, and knowledge of developmental biology and organogenesis, which allow for manipulation of neural stem cells in the bowel wall for replacement therapy.

\section{F1. Objectives}

- Determine specific gene profiles in tissues that suffer ICC or neuron loss or in tissues in the process of losing these elements and develop a molecular test to detect these pathological changes.

F1. Comments Our understanding of ICC pathology is still in its infancy, but a picture is beginning to emerge that many disparate motility disorders result in loss of ICC. Mechanisms responsible for the demise of ICC are poorly understood. One approach is to make large- 
scale screenings of genetic changes that occur in tissues undergoing loss of ICC and investigate whether there are common genetic fingerprints that either produce or result from loss of ICC. Pathophysiological models coupled with genomics analyses may offer the opportunity to discover why the ICC is vulnerable in apparently disparate motor disorders and how to recover ICC networks in disease organs. From these studies, it may be possible to determine specific gene profiles that occur in tissues that have suffered ICC loss, or in tissues in the process of losing ICC. This information may allow scientists to design specialized tests that reliably evaluate the status of ICC networks from biopsy material. Development of a molecular test that could pick up pathological changes more reliably and at earlier time points may be highly beneficial as we may be able to halt the degeneration and minimize damage and loss of function.

\section{F2. Objectives}

- Develop neural imaging techniques to correlate individual circuits identified with symptom production in IBS patients and establish correlation with distinct genotypes through such approaches as a genome-wide search for polymorphisms and haplotypes.

F2. Comments Advances in neuroimaging studies of the CNS should facilitate our efforts to identify distinct brain circuits responsible for various GI functions and pain perception. Once this information becomes available, we should characterize the signalling systems and receptors within these neural circuits using PET ligand imaging in rodents and in humans. Ultimately, we should be able to correlate the individual circuits identified with symptom production in patients (intermediate phenotypes) and establish correlation with distinct genotypes that include a genome-wide search for polymorphism and haplotypes. Ultimately, we need to be able to characterize fully the pathomechanisms underlying symptom generation in common functional and motility disorders, including identification of genetic and early environmental influences and natural course of the disease. This should lead to more effective prevention and therapeutic intervention.

\section{F3. Objectives}

- Develop neuron replacement therapy to guide the growth of enteric axons to their targets as a therapy for neural degenerative disease of the ENS.

F3. Comments In addition to the study of in vivo migrating behaviour of ENS precursor cells and the characterization of guidance molecules on axonal growth. Information on local tissue microenvironments (niches) that maintain and regulate stem cells has begun to emerge. ${ }^{69}$ Gut NCSCs may lie dormant and can then be activated following injury. This appears to occur under tightly controlled niches. Niches changes may occur with age and contribute to tissue ageing. Research in this arena may provide new preventive or therapeutic approaches to degenerative neuron loss of the ENS. Currently, most of the information on the environmental mechanisms that regulate stem cell function is obtained from invertebrate systems where stem cells from a variety of tissues can be imaged and genetically modified. Technical advances will be required in the vertebrate systems to improve imaging of stem cells at a single cell resolution. This will allow the verification of the true identity of the cell studied based on the markers it expresses. It is also important to systematically test mechanisms that are proposed to regulate stem cell maintenance using genetics. A better understanding of the mechanisms that regulate stem cell maintenance will facilitate development of new strategies to promote tissue regeneration after injury and to maintain stem cell activity during ageing.

\section{F4. Objectives}

- Characterize the molecular, cellular and behavioural mechanisms that link changes of stored body fat to adaptive adjustments of feeding behaviours by defining the diverse blood-borne and affective neural signals that transmit information regarding nutrient status and energy stores to the brain where it is integrated with cognitive, visual, olfactory and taste cues.

F4. Comments Leptin is produced in white adipose tissue and circulates in proportions with the adipose mass, increasing in plasma as animals and humans become obese and decreasing in times of food deprivation. Thus, leptin provides a signal of the availability of the body's energy stores. Another adiposity factor important for the long-term control of energy balance is insulin which is also secreted in proportion to body mass. Both leptin and insulin interacts with same hypothalamic circuits to affect overall energy balance. Animal and human studies suggest that insensitivity to leptin and insulin may be responsible for obesity in some individuals. ${ }^{70-71}$ The cellular and molecular mechanisms responsible for this phenomenon await further investigation. With a better understanding of the hypothalamic systems involved in feeding control, we can begin to identify the distinct neural circuits 
responsible for within-meal and across-meal signalling. There is still much to be learned about how various satiety peptides, neural signals and micronutrients interact at the level of the hypothalamus to modulate food intake and energy homeostasis. At a molecular level, phosphorylation of AMP-activated protein kinase (AMPK) in the hypothalamus appears to play a critical role in regulating feeding behaviour. ${ }^{72}$ It is conceivable that AMPK may act as a physiological energy sensor within the hypothalamus, and that alterations in AMPK may be a common final readout of variety of treatments that act within the basal hypothalamus to affect energy balance. This is an important area for further investigation.

The molecular mechanism linking adiposicity to overnutrition remains unknown. Recent literature suggests that gastric inhibitory polypeptide (GIP) is a key molecule linking overnurtition to obesity. Excessive fat intake induces hypersecretion of GIP, which increases nutrient update and triglyceride accumulation at the adipose sites. This can readily cause obesity and hyperinsulinaemia. Hyperinsulinaemia will further increase nutrient uptake into adipose sites, completing a vicious cycle of developing adiposicity. Further studies should be performed to explore the possibility of GIP abnormalities in patients with overnutrition and obesity.

\section{CONCLUDING REMARKS}

Advances in the molecular biology sciences and proteomics represent an unprecedented opportunity for us to understand key biological pathways pertaining to the neuroendocrine system of the GI tract. The unravelling of the complex signalling between diverse cells in the ENS provides a cellular and molecular basis for understanding many disorders in the ENS. In the past, the quest for therapeutic targets and lead molecules has concentrated on neurotransmitters, hormones and their receptors. However, much more is now known about ion channels and intracellular messengers that control excitability of enteric neurons, the effector cells they innervate, and the enteroendocrine cells that activate enteric primary afferent neurons. The ion channels and intracellular messengers may provide new and viable targets. The neurobiology of brain-gut interactions has become better characterized, providing the necessary conceptual framework for developing new treatments of functional GI diseases and motility disorders. There is renewed and expanded interest in the role of the GI tract in the regulation of satiety and energy homeostasis. Increased understanding of the mechanism governing nutrient sensing and peptide secretion by enteroendocrine cells allows investigators to exploit these pathways in the development of new agents to combat obesity and diabetes. A better understanding of the molecular mechanisms leading to disease and age-related apoptotic cell death provides hope for preventive and/or regenerative therapy. Impressive recent advances in the identification of molecules that are involved in regulating the differentiation, maturation and guidance to targets of enteric neurons may provide the ways to restitute neuropathic deficiencies that lead to disordered GI function. The Commission identified a set of high priority goals for which coordinated research efforts would accelerate progress across the digestive disease research field including neurogastroenterology. The stage is set to put that knowledge to advantage in improving the treatment of patients with GI disorders.

\section{ACKNOWLEDGMENTS}

The author would like to thank the following individuals for their insightful comments and suggestions during the preparation of this recommendation for the National Commission on Digestive Diseases, sponsored by the National Institute of Diabetes and Digestive and Kidney Diseases: Drs Gianrico Farrugia, Lee Kaplan, Emeran Mayer, Kent Sanders and Michael Wolfe.

\section{REFERENCES}

1 Furness JB. The enteric nervous system: normal functions and enteric neuropathies. Neurogastroenterol Motil 2008; 20(Suppl. 1): 32-8.

2 Burns AJ, Lomax AZ, Torihashi S, Sanders KM, Ward SM. Interstitial cells of Cajal mediate inhibitory transmission in the stomach. Proc Natl Acad Sci USA 1996; 93: 12008 13.

3 Clerc N, Gola M, Vogalis F, Furness JB. Controlling the excitability of IPANs: a possible route to therapeutics. Curr Opin Pharmacol 2002; 2: 657-64.

4 Mayer EA, Marvizon JC. Neurokinin 3 receptors in the gut: a new target for treatment of visceral pain? Gastroenterology 1999; 116: 1250-2.

5 Buno L, Fioramonti J, Garcia-Villar R. Pathobiology of visceral pain: molecular mechanisms and therapeutic implications. Visceral afferent pathways: a source of new therapeutic targets for abdominal pain. Am J Physiol 2000; 278: G670-6.

6 Bertrand PP, Bornstein JC. ATP as a putative sensory mediator: activation of intrinsic sensory neurons of the myenteric plexus via P2X receptors. I Neurosci 2002; 22: 4767-75.

7 Bertrand PP, Junze WAA, Furness JB, Bornstein JC. The terminals of myenteric intrinsic primary afferent neurons on the guinea-pig ileum are excited by 5 -hydroxytrypta- 
mine acting at 5-hydroxytryptamine-3 receptors. Neuroscience 2000; 101: 459-69.

8 Kunze WA, Clerc N, Bertrand PP, Furness JB. Contractile activity in intestinal muscle evokes action potential discharge in guinea-pig myenteric neurons. J Physiol (Lond) 1999; 517: 547-61.

9 Kunze WA, Clerc N, Furness JB, Gola M. The soma and neuritis of primary afferent neurons in the guinea-pig intestine respond differentially to deformation. I Physiol (Lond) 2000; 526: 375-85.

10 Starodub AM, Wood JD. Histamine suppresses A-type potassium current in myenteric neurons from guinea pig small intestine. J Pharmacol Exp Ther 2000; 294: 555-61.

11 Alex G, Kunze WAA, Furness JB, Clerc N. Comparison of the effects of neurokinin-3 receptor blockade on two forms of slow synaptic transmission in myenteric $\mathrm{AH}$ neurons. Neuroscience 2001; 104: 263-9.

12 Linden DR, Manning BP, Bunnett NW, Mawe GM. Agonists of proteinase-activated receptor 2 excite guinea pig ileal myenteric neurons. Eur I Pharmacol 2001; 431: 311-4.

13 Wada-Takahashi S, Tamura K. Actions of reactive oxygen species on $\mathrm{AH} /$ type 2 myenteric neurons in guinea pig distal colon. Am J Physiol 2000; 279: G893-902.

14 Kelles A, Janssens J, Tack J. IL- $1 \beta$ and Il-6 excite neurones and suppress cholinergic neurotransmission in the myenteric plexus of the guinea pig. Neurogastroenterol Motil 2000; 12: 531-8.

15 Kruger GM, Mosher JT, Bixby S, Joseph N, Iwashita T, Morrison SJ. Neural crest stem cells persist in the adult gut but undergo changes in self-renewal, neuronal subtype potential, and factor responsiveness. Neuron 2002; 35: 657-69.

16 Bixby S, Kruger GM, Mosher JT, Joseph NM, Morrison SJ. Cell-intrinsic differences between stem cells from different regions of the peripheral nervous system regulate the generation of neural diversity. Neuron 2002; 35: 64356.

17 Gianina S, Grider JR, Cresswell J et al. GDNF availability determines enteric neuron number by controlling precursor proliferation. Development 2003; 130: 2187-98.

18 Moore MW, Klein RD, Farinas I et al. Renal and neuronal abnormalities in mice lacking GDNF. Nature 1996; 382: 76-9.

19 Sanchez MP, Silos-Santiago I, Frisen J et al. Renal agenesis and the absence of enteric neurons in mice lacking GDNF. Nature 1996; 382: 70-3.

20 Bush TG, Savidge TC, Freeman TC et al. Fulminant jejuno-ileitis following ablation of enteric glia in adult transgenic mice. Cell 1998; 93: 189-201.

21 Comet A, Savidge TC, Cabarrocas J et al. Enterocolitis induced by autoimmune targeting of enteric glial cells: a possible mechanism in Crohn's disease? Proc Natl Acad Sci USA 2001; 98: 13306-11.

22 Steinkamp M, Geerling I, Seufferlein T et al. Glial-derived neurotrophic factor regulates apoptosis in colonic epithelial cells. Gastroenterology 2003; 124: 1748-57.

23 Lomax AE, Mawe GM, Sharkey KA. Synaptic facilitation and enhanced neuronal excitability in the submucosal plexus during experimental colitis in guinea-pig. I Physiol 2005; 564: 863-75.

24 Collins SM, Blennerhassett PA, Blennerhassett MG, Vermillion DL. Impaired acetylcholine release from the myenteric plexus of Trichinella-infected rats. Am $J$ Physiol 1989; 257: G898-903.

25 Davis KA, Masella J, Blennerhassett MG. Acetylcholine metabolism in the inflamed rat intestine. Exp Neurol 1998; 152: 251-8.

26 Main C, Blennerhassett P, Collins SM. Human recombinant interleukin 1 beta suppresses acetylcholine release from rat myenteric plexus. Gastroenterology 1993; 104: 1648-54.

27 Sturiale S, Barbara G, Qiu B et al. Neutral endopeptidase (EC 3.4.24.11) terminates colitis by degrading substance P. PNAS 1999; 96: 11653-8.

$28 \mathrm{Hu} \mathrm{W}$, Li F, Murthy KS. Interleukin- $1 \beta$ stimulates expression of NADPH oxidases NOX1 and NOX4 in colonic smooth muscle: mediation by NF- $\kappa \mathrm{B}$ and differential modulation by c-jun kinase and ERK1/2. Gastroenterology 2006; 130: A505.

29 Murthy KS, Mahavadi S, Makhlouf GM. Identification of the signaling mechanisms that mediate inhibition of colonic muscle contraction by interleukin-1 $\beta$ : up-regulation of RGS4 and down-regulation of CPI-17. Gastroenterology 2005; 128: A497.

30 Horowitz B, Ward SM, Sanders KM. Cellular and molecular basis for electrical rhythmicity in gastrointestinal muscles. Annu Rev Physiol 1999; 61: 19-43.

31 Sanders KM, Koh SD, Ward SM. Interstitial cells of Cajal as pacemakers in the gastrointestinal tract. Annu Rev Physiol 2006; 68: 307.

32 Liu X, Rusch NJ, Striessnig J, Sarna SK. Down-regulation of L-type calcium channels in inflamed circular smooth muscle cells of the canine colon. Gastroenterology 2001; 120: 480-9.

33 Thuneberg L, Peters S. Toward a concept of stretch-coupling in smooth muscle. I. Anatomy of intestinal segmentation and sleeve contractions. Anat Rec 2001; 262: 110-24.

34 Lu G, Qian X, Berezine I, Telford GL, Huizinga JD, Sarna SK. Inflammation modulates in vitro colonic myoelectric and contractile activity and interstitial cells of Cajal. Am J Physiol 1997; 273: G1233-45.

35 Cheng L, Cao W, Behar J et al. Acid-induced release of platelet-activating factor by human esophageal mucosa induces inflammatory mediators in circular smooth muscle. I Pharm Exp Ther 2006; 319: 117-26.

36 Gershon MD. Plasticity in serotonin control mechanisms in the gut. Curr Opin Pharmacol 2003; 3: 600-7.

37 Giatzle J, Sternini C, Robin C et al. Expression of 5HT3 receptors in the rat gastrointestinal tract. Gastroenterology 2002; 123: 217-26.

38 Grider JR, Kuemmerle JF, Jin JG. 5HT released by mucosal stimuli initiates peristalsis by activating $5 \mathrm{HT}_{4} / 5 \mathrm{HT}_{\mathrm{IP}}$ receptors on sensory CGRP neurons. Am I Phsyiol 1996; 270: G778-82.

39 Prims NH, Akkermans LM, Lefebvre RA, Schuurkes JA. $5 \mathrm{HT}_{4}$ receptors on cholinergic nerves involved in contractility of caine and human large intestinal longitudinal muscle. Br I Pharmacol 2000; 131: 927-32.

40 Tache Y, Bonaz B. Corticotropin-releasing factor receptors and stress-related alterations of gut motor function. I Clin Invest 2007; 117: 33-40.

41 Tache Y, Perdue MH. Role of peripheral CRF signaling pathways in stress-related alterations of gut motility and 
mucosal function. Neurogastroenterol Motil 2004; 16(Suppl. 1): 1-6.

42 Kihara N, Fujimura M, Yamamoto I et al. Effects of central and peripheral urocortin on fed and fasted gastroduodenal motor activity in conscious rats. Am I Physiol Gastrointest Liver Physiol 2001; 280: G406-19.

43 Martinez V, Wang L, Rivier JE, Vale W, Tache Y. Differential actions of peripheral corticotrophin-releasing factor (CRF), urocortin II, and urocortin III on gastric emptying and colonic transit in mice: role of CRF receptor subtypes 1 and 2. J Pharmacol Exp Ther 2002; 301: 611-7.

44 Million $M$ et al. Human urocortin II, a new CRF-related peptide, displays selective $\mathrm{CRF}_{2}$-mediated action on gastric transit in rats. Am J Physiol Gastrointest Liver Physiol 2002; 282: G34-40.

45 Martinez V, Tache Y. CRF1 receptors as a therapeutic target for irritable bowel syndrome. Curr Pharm Des 2006; 12: 4071-88.

46 Gravanis A, Margioris AN. The corticotrophin-releasing factor family of neuropeptide in inflammation: potential therapeutic applications. Curr Med Chem 2005; 12: $1503-$ 12.

47 Chalazonitis A, Rothman TP, Chen J, Gershon MD. Agedependent differences in the effects of GDNF and NT-3 on the development of neurons and glia from neural crestderived precursors immunoselected from the fetal rat gut: expression of GFRalpha-1 in vitro and in vivo. Dev Biol 1998; 204: 385-406.

48 Hearn CJ, Murphy M, Newgreen D. GDNF and ET-3 differentially modulate the numbers of avian enteric neural crest cells and enteric neurons in vitro. Dev Biol 1998; 197: 93-105.

49 Heuckeroth RO, Lampe PA, Johnson EM, Milbrandt J. Neurturin and GDNF promote proliferation and survival of enteric neuron and glial progenitors in vitro. Dev Biol 1998; 200: 116-29.

50 Taraviras S, Marcos-Gutierrez CV, Durbec P et al. Signalling by the RET receptor tyrosine kinase and its role in the development of the mammalian enteric nervous system. Development 1999; 126: 2785-97.

51 Iwashita T, Kruger GM, Pardal R, Kiel MJ, Morrison SJ. Hirschsprung disease is linked to defects in neural crest stem cell function. Science 2003; 301: 972-6.

52 Bordeaux MC, Forcet C, Granger L et al. The RET protooncogene induces apoptosis: a novel mechanism for Hirschsprung disease. EMBO J 2000; 19: 4056-63.

53 Mehlen P, Bredesen DE. The dependence receptor hypothesis. Apoptosis 2004; 9: 37-49.

54 Tsai YH, Gariepy CE. Dynamic changes in the proximal gut neural crest stem cell population are associated with successful development of the distal enteric nervous system in rats. Pediatr Res 2005; 58: 636-43.

55 Bondurand N, Natarajan D, Barlow A, Thapar N, Pachnis $\mathrm{V}$. Maintenance of mammalian enteric nervous system progenitors by Sox10 and Endothelin-3 signalling. Development 2006; 133: 2075-86.

56 Dyer J, Salmon KS, Zibrik L, Shirazi-Beechey SP. Expression of sweet taste receptors of the T1R family in the intestinal tract and enteroendocrine cells. Biochem Soc Trans 2005; 33: 302-5.
57 Wu SV, Rozengurt N, Yang M, Young SH, Sinnett-Smith J, Rozengurt E. Expression of bitter taste receptors of the T2R family in the gastrointestinal tract and enteroendocrine STC-1 cells. Proc Natl Acad Scie USA 2002; 99: 2392-7.

58 Hofer D, Puschel B, Drenckhahn D. Taste receptorlike cells in the rat gut identified by expression of alpha-gustducin. Proc Natl Acad Sci USA 1996; 93: 66314.

59 Chen MC, Wu V, Reeve JR, Rozengurt E. Bitter stimuli induce $\mathrm{Ca}^{2+}$ signaling and $\mathrm{CCK}$ release in enteroendocrine STC-1 cells: role of L-type voltage-sensitive $\mathrm{Ca}^{2+}$ channels. Am I Physiol Cell Physiol 2006; 291: C726-39.

60 Rozengurt N, Wu SV, Chen MC, Huang C, Sternini C, Rozengurt E. Colocalization of the alpha-subunit of gustducin with PYY and GLP-1 in L cells of human colon. Am I Physiol Gastrointest Liver Physiol 2006; 291: G792802.

61 Margolskee RF. Molecular biology of taste perception. Diabetes 2006; 55(Suppl. 1): R1293-307.

62 Grider JR. Identification of neurotransmitters regulating intestinal peristaltic reflex in humans. Gastroenterology 1989; 97: 1414-9.

63 Anlauf M, Schäfer MK-H, Eiden L, Weihe E. Chemical coding of the human gastrointestinal nervous system: cholinergic, VIPergic, and catecholaminergic phenotypes. I Comp Neurol 2003; 459: 90-111.

64 Mayer EA, Naliboff BD, Craig ADB. Neuroimaging of the brain-gut axis: from basic understanding to treatment of functional GI disorders. Gastroenterology 2006; 131: 1925-42.

65 Metz H, Morgan V, Tanner G et al. Regional cerebral activation in irritable bowel syndrome and control subjects with painful and non painful rectal distension. Gastroenterology 2000; 118: 842-8.

66 Meyer-Lindenberg A, Weinberger DR. Intermediate phenotypes and genetic mechanisms of psychiatric disorders. Nat Rev Neurosci 2006; 7: 818-27.

67 Bergstrom M, Hargreaves RJ, Burns HD et al. Human positron emission tomography studies of brain neurokinin 1 receptor occupancy by aprepitant. Biol Psychiatr 2004; 55: 1007-12.

68 Cummings DE, Overduin J. Gastrointestinal regulation of food intake. J Clin Invest 2008; 117: 13-28.

69 Morrison SJ, Spradling AC. Stem cells and niches: mechanisms that promote stem cell maintenance throughout life. Cell 2008; 132: 598-611.

70 El-Haschimi K, Pierroz DD, Hileman SM, Bjorbaek C, Flier JS. Two defects contribute to hypothalamic leptin resistance in mice with diet-induced obesity. J Clin Invest 2000; 105: 1827-32.

71 Kaiyala KJ, Prigeon RL, Kahn SE, Woods SC, Schwartz MW. Obesity induced by a high-fat diet is associated with reduced brain insulin transport in dogs. Diabetes 2000; 49: 1525-33.

72 Kim EK, Miller I, Aja S et al. C75, a fatty acid synthase inhibitor, reduces food intake via hypothalamic AMPactivated protein kinase. I Biol Chem 2004; 279: 19970-6. 